\title{
Experiencia internacional en relación al reposo médico y a la protección de los trabajadores frente a la incapacidad transitoria de generar ingresos: referente para el caso chileno
}

\author{
MARCOS VERGARA I.
}

\section{International policies towards sick leave. A referent to analyze the Chilean situation}

This is a comparative review of sick leave and wage replacement policies among countries of the Organization for Economic Co-operation and Development (EOCD), an organism that Chile joined recently. This review also deals with the main transformations applied to the system to improve its efficiency and the variables that explain such changes. The Chilean system is analyzed from the perspective of substitution rates, waiting time and protection length. According to these parameters, Chile is located below countries such as Scandinavian countries, that provide a universal protection, and over those countries that provide a focalized protection according to worker's needs.

(Rev Med Chile 2011; 139: 495-504).

Key words: Salaries and fringe benefits; Sick leave; Social security.

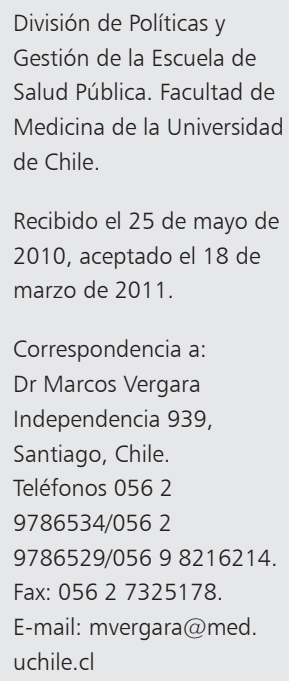

S e recurre a la experiencia internacional para establecer la posición relativa de Chile en materia de reposo médico y reemplazo de ingresos de los trabajadores (subsidio por incapacidad laboral). Conocer otras realidades es útil para actualizar estas políticas públicas, razón por la cual las descripciones se realizan en toda su riqueza y diversidad.

No es el propósito de esta revisión poner un foco en los problemas que afectan al seguro en Chile, los que han venido formando parte del debate público, asociados a la vulnerabilidad y obsolescencia del seguro y a visiones de mundo contrapuestas y muchas veces en conflicto.

Aedo y otros ${ }^{1}$ abordan las dificultades que ofrece el camino de la comparación, que no sólo encuentra problemas por la diversidad de los sistemas de protección entre países (si cuentan o no con protección especial en problemas de salud asociados al trabajo, si se protege o no a la maternidad dentro del seguro, si es posible ir más allá del empleo formal), sino también por la calidad de los reportes ${ }^{2}$.

Las comparaciones están afectadas por la proporción de población económicamente activa y la estructura por edad y sexo de la misma: la población chilena es más joven y con menor porcentaje de mujeres en el mercado laboral respecto de países desarrollados y es más vieja y con mayor porcentaje de mujeres en el trabajo que en algunos países de América Latina. Este tipo de seguros suele estar vinculado al empleo formal de las economías, de modo tal que su cobertura estará determinada por el grado de formalidad del mercado del trabajo.

También las comparaciones se afectan por la presencia de sistemas de seguridad social más robustos en países desarrollados que, a consecuencia de ello, no producen sobre el sistema de 
subsidios por incapacidad laboral presiones para su uso frente a otras necesidades, como sí ocurriría en Chile $^{3}$ a pesar de que su sistema de seguridad social establecido es considerado "comprehensivo" por la Organización Internacional del Trabajo ${ }^{4}$. Producto de lo anterior, algunos autores afirman que el sistema en Chile es generoso en la protección que brinda ${ }^{5}$ mientras otros consideran que sus niveles de gasto y beneficios son moderados ${ }^{1,3}$, si se consideran carencias y topes que implican una tasa de reemplazo promedio del $75 \%$.

\section{El referente OECD (Organización para la Cooperación y el Desarrollo Económico)}

\section{Validez del referente para Chile}

Este referente OECD es válido por cuanto Chile heredó precozmente la tradición de la seguridad social alemana -el modelo de Bismarck-gracias al impulso de González Cortés en un contexto sociopolítico comparable ${ }^{5,7,8}$, así como la aproximación Universalista propuesta por Beveridge, basada en ciudadanía.

El seguro social para sustituir ingresos por enfermedad se ha mantenido en Chile bajo el esquema original, con tendencia a mejorar cobertura y tasa de reemplazo y algunas modificaciones en su financiamiento, ocurridas en el contexto de transformaciones del sistema previsional de pensiones y de salud, durante el gobierno militar.

Desde el punto de vista del sector salud como sector económico ${ }^{4}$, los referentes de las últimas décadas han sido, en el gobierno militar, el modelo norteamericano de seguros privados de salud y oferta privada de servicios médicos y, durante los gobiernos de la Concertación, el modelo inglés de la separación de funciones y creación de cuasi mercados ${ }^{9}$. También ha sido importante el modelo holandés para la reflexión intelectual de inspiración universalista de especialistas interesados en el desarrollo de los seguros previsionales privados.

\section{Los países de la OECD}

\section{Perspectiva amplia de análisis}

El análisis de los sistemas de seguridad social no remitiría exclusivamente a dimensiones clásicas del Welfare State. Si seguimos a Castles ${ }^{10}$, las regulaciones sobre el mercado laboral, la promoción del empleo a discapacitados, las políticas de salarios, las de vivienda y las de subsidios para servicios básicos, configuran un espacio amplio donde se resuelven los estados de necesidad, al cual los esquemas de Welfare State contribuyen. El resultado sería un alto grado de heterogeneidad en los sistemas de protección social ${ }^{11}$. Según Förster ${ }^{12}$ existiría un movimiento de beneficios asociados a discapacidad -transitoria o permanente, total o parcial- hacia programas soportados en el mercado del trabajo, con incentivos sobre empleadores y empleados.

En Chile, entre 1990 y 2010, los Presidentes Aylwin, Frei, Lagos y Bachellet consolidaron políticas públicas con enfoque de derechos e instalaron un discurso y una práctica relativa a un eje amplio de protección social (que se asimila al concepto de seguridad social en el sentido de ser provista -la protección-por el mismo -el sistema de seguridad social- $)^{4}$, expresado en programas como el plan de garantías explícitas en salud ${ }^{14}$, "Chile Crece Contigo"13 y la reforma al sistema de pensiones que amplió su cobertura respecto de los trabajadores con empleo formal ${ }^{15}$.

\section{Tipos de sistemas}

En los países de la OECD se distinguen sistemas de seguridad social de corte universalista, como los escandinavos, otros que se basan en categorías laborales, como el alemán y, por último, aquellos donde los beneficios están condicionados a las necesidades, como Australia, Nueva Zelandia y Reino Unido ${ }^{16,17}$. Si bien los países están desarrollando enfoques diversos para enfrentar estados de necesidad, de cara a transformaciones demográficas, según Clasen ${ }^{17}$, a pesar de vivir el Welfare State momentos de crisis, no se apreciaría en Alemania, Holanda y en los países escandinavos un movimiento alejado de los esquemas tradicionales, como sí ocurrió en el Reino Unido y como se debate hoy en España en cuanto al seguro de cesantía y el sistema de pensiones.

Clasen ${ }^{17}$ ordena su análisis en función de categorías identificadas en la Tabla 1. Sin embargo, la claridad de la tabla no se refleja tan nítidamente en la realidad, donde los modelos se mezclan, no hay tendencias transnacionales y los principios no gobiernan ex-antes las decisiones.

Superando la taxonomía que refiere a modelos social-demócratas, liberales y conservadores de seguridad social, Korpi ${ }^{18}$ distingue 5 tipos: i) el focalizado, estatal y orientado a garantizar mínimos según la situación económica; ii) el voluntario 
Política internacional en reposo médico y situación en Chile - M. Vergara

Tabla 1. Correspondencias típicas entre definición de necesidades, principios de justicia y derechos sociales, regímenes y esquemas de seguridad social

\begin{tabular}{|c|c|c|c|}
\hline $\begin{array}{l}\text { Definición de } \\
\text { necesidad }\end{array}$ & $\begin{array}{l}\text { Mínimo estandar de } \\
\text { sobrevivencia }\end{array}$ & $\begin{array}{l}\text { Recuperación del } \\
\text { estándar perdido }\end{array}$ & $\begin{array}{l}\text { Standard para nivel } \\
\text { de vida razonable }\end{array}$ \\
\hline $\begin{array}{l}\text { Principios Generales de } \\
\text { justicia (re) distributiva }\end{array}$ & Necesidad & Equidad & Igualdad \\
\hline $\begin{array}{l}\text { Términos alternativos para } \\
\text { principios en política social }\end{array}$ & Selectividad & $\begin{array}{l}\text { Reciprocidad } \\
\text { Régimen contributivo } \\
\text { Proporcionalidad } \\
\text { Equivalencia } \\
\text { Lógica de Seguro }\end{array}$ & $\begin{array}{l}\text { Universalismo } \\
\text { Incondicionalidad }\end{array}$ \\
\hline Tipos de derechos sociales & Basado en Pobreza & Basado en reciprocidad & Basado en ciudadanía \\
\hline Régimen de Bienestar & Liberal & Conservador & Social-demócrata \\
\hline $\begin{array}{l}\text { Tradición de la seguridad } \\
\text { social }\end{array}$ & Asistencia Social & Seguro Social & $\begin{array}{l}\text { Beneficios categóricos o } \\
\text { universales }\end{array}$ \\
\hline $\begin{array}{l}\text { Objetivo general de la segu- } \\
\text { ridad social }\end{array}$ & Reducción de la pobreza & Preservación del status & Promoción del buen vivir \\
\hline $\begin{array}{l}\text { Características típicamente } \\
\text { Asociadas }\end{array}$ & $\begin{array}{l}\text { Fondos estatales } \\
\text { Beneficios mínimos } \\
\text { Medición de necesidades }\end{array}$ & $\begin{array}{l}\text { Fondo contributivo } \\
\text { (impuesto al trabajo) } \\
\text { Beneficios relativos al } \\
\text { ingreso (Bismarck) o } \\
\text { Beneficios universales } \\
\text { (Beveridge) } \\
\text { Contribución en función } \\
\text { de riesgos }\end{array}$ & $\begin{array}{l}\text { Fondos estatales } \\
\text { Beneficios universales } \\
\text { Test de ciudadanía }\end{array}$ \\
\hline
\end{tabular}

Fuente: Clasen, J. \& Wim Van Oorschot. Changing Principles in European Social Security. European Journal of Social Security. 4(2002), página 94.

subsidiado, administrado por sus miembros, subsidiado por el estado y con beneficios proporcionales a ingresos, relativamente bajos; iii) el corporativo, basado en aportes de miembros correspondientes a alguna categoría laboral, con beneficios proporcionales a ingresos y administrados con participación de empleados, empleadores y, eventualmente, el estado; iv) el básico, que establece un piso de beneficios para todos sobre la exclusiva base de su ciudadanía, con coberturas universales y; v) el abarcativo, que combina universalidad de cobertura de la seguridad básica con tasas de reemplazo de ingresos altas, como las del modelo corporativo, administrado por entidades públicas.

Según Kangas ${ }^{11}$, entre los años 1930 y 1995, los países europeos mejoraron su posición en cuanto a cobertura poblacional y tasa de reemplazo -porcentaje de la remuneración reemplazada-. Destacan por encima del promedio Alemania, con apreciable mejoría en la tasa de reemplazo y Noruega, con apreciable mejoría en la cobertura y, por debajo, Reino Unido, con especial deterioro de cobertura. Al momento de producir ajustes, las soluciones predominantemente estatales tienen mayor capacidad de adaptación que las corporativas, por estar estas últimas atadas a intereses y por ser las primeras de propiedad del cuerpo social, que resuelve sus asuntos en la arena política ${ }^{11}$.

$\mathrm{Korpi}^{18}$, por su parte, intenta demostrar que la lógica de las clases importa para explicar el comportamiento del Welfare State, en particular en momentos de estrechez, recurriendo a la idea de activos personales para aprovechar la estructura de oportunidades que se presenta en la sociedad ${ }^{19}$, ampliando la aproximación marxista hacia formas de capital como prestigio y capital cultural. Según Korpi ${ }^{18}$, el Welfare State sigue siendo arena para el despliegue y resolución de conflictos entre clases, específicamente entre empleadores y empleados y, al mismo tiempo, es un output de los mismos. El autor refuta afirmaciones que subyacen a la idea del fin de las clases en el Welfare State: i) que la 
mirada de clases desconoce el aporte de los empleadores; ii) que los políticos, que representarían intereses de clases, toman distancia del tema en períodos de estrechez y; iii) que nuevos grupos cobrarían importancia, como pensionados, discapacitados y beneficiarios de los sistemas de salud.

Importante es la tolerancia de la sociedad con sistemas de asignación de beneficios basados en evaluación de necesidades (focalizados). Kangas ${ }^{16}$ aborda esto en países escandinavos, con coberturas que trascienden a los trabajadores. Con una tabla de doble entrada para las variables participación en el financiamiento -alta, baja- y probabilidad de recibir beneficios -alta, baja-, distingue cuatro grupos de interés, dos neutros -el grupo que no aporta y no recibe y el grupo que aporta y recibe- $y$ dos interesados, uno en contra del esquema -que aporta sin recibir- y uno a favor -que recibe sin aportar-.

Existen, sin embargo, variables políticas, que conducen a resultados no explicables por la racionalidad del interés individual, como socialdemócratas preocupados por la estigmatización social, que aceptan cortes solo en la parte alta de la pirámide de ingresos, o trabajadores que aunque les conviene la asignación basada en necesidades, les resulta políticamente impropio apoyarla. A esto se agregan los tipos de necesidades, pues no es igual para salud o pensiones, donde se está más a favor de un acceso universal, que para vivienda, donde ayudar sobre una base de necesidades es aceptable.

Al preguntar Kangas ${ }^{16}$ si acaso debieran aumentarse los criterios de asignación basados en necesidades, la respuesta es que mientras mayores son los ingresos, menor es el interés de los grupos por el sistema basado en necesidades, con diferencias estadísticamente significativas.

\section{El desempeño de los sistemas}

La pregunta que surge en el espacio de las políticas públicas es acerca de cuán generoso cada sistema sería y aparece la dificultad de ilustrar el fenómeno utilizando el gasto social, según Ade$\mathrm{ma}^{20}$, por dos razones: i) dificultad para capturar los gastos que se desprenden de ventajas tributarias otorgadas a empresas que destinan recursos a programas sociales, generándose discrepancias entre el presupuesto y el gasto efectivo y, ii) mucho gasto social ocurre fuera del presupuesto público, financiado con contribuciones privadas a partir de mandatos públicos.

Además corrige la línea base de gasto social deduce los impuestos al gasto social y agrega gasto mandatado e inducido por ventajas tributariaspara una serie de países, según se ilustra en Tabla 2.

Según Korpi ${ }^{18}$, las tradicionales medidas de gasto público o fiscal en seguridad social como porcentaje del producto, presenta defectos que se agudizan en períodos con alto desempleo o bajo crecimiento del producto, donde generan una ilusión contra-cíclica. La propuesta del autor, a partir de una conceptualización que incluiría políticas de empleo y otras que afectan el mercado laboral, es el Social Citizenship Indicator Program-SCIP-, del que se desprende la Tasa Neta de Reemplazo de ingresos, después de impuestos y cotizaciones. Korpi ${ }^{18}$ estudia tres seguros que protegen pérdidas de ingresos: i) por enfermedad, ii) por accidentes del trabajo y, iii) por desempleo. La Tasa Neta de Reemplazo es calculada para años seleccionados entre 1930 y 1995. En el caso del seguro por enfermedad se observa primero un crecimiento de la tasa, con una declinación del orden de unos 5 puntos porcentuales entre 1975 y 1995, de 70\% a $65 \%$, declinación significativa en el Reino Unido,

Tabla 2. Gasto social y gasto social ajustado por adema, expresado como porcentaje del PGB a costo de factores (datos de 1995)

\begin{tabular}{|c|c|c|c|c|c|c|c|c|c|c|c|}
\hline & $\begin{array}{l}\text { Aus- } \\
\text { tralia }\end{array}$ & $\begin{array}{c}\text { Cana- } \\
\text { da }\end{array}$ & $\begin{array}{l}\text { Dina- } \\
\text { marca }\end{array}$ & $\begin{array}{c}\text { Fin- } \\
\text { landia }\end{array}$ & $\begin{array}{l}\text { Ale- } \\
\text { mania }\end{array}$ & $\begin{array}{l}\text { Irlan- } \\
\text { da }\end{array}$ & Italia & $\begin{array}{l}\text { Holan- } \\
\text { da }\end{array}$ & Suecia & UK & USA \\
\hline $\begin{array}{l}\text { Gasto } \\
\text { social }\end{array}$ & 20,3 & 20,8 & 37,6 & 35,7 & 30,4 & 21,8 & 26,5 & 30,1 & 36,4 & 25,9 & 17,1 \\
\hline $\begin{array}{l}\text { Gasto } \\
\text { social } \\
\text { ajustado }\end{array}$ & 21,6 & 21,2 & 24,4 & 25,7 & 27,7 & 18,7 & 22,3 & 25,0 & 27,0 & 26,0 & 24,5 \\
\hline
\end{tabular}

Adema, W. Revisiting Real Social Spending Across Countries: a Brief Note. OECD Economics Studies 2000; 30: $192-197$. Extractado de Tabla 1. 
donde retorna a niveles pre-Beveridge, del orden de 20\%, desde 63,4\% en 1975 .

$\mathrm{Korpi}^{18}$ identifica y mide la importancia de variables que operarían como independientes y que explicarían el comportamiento de una variable dependiente representada por el número de recortes mayores a la Tasa de Reemplazo de los ingresos para cualquiera de los tres tipos de seguros, por cada 100 años-país de exposición al riesgo. Las variables fueron: tasas de desempleo, tipo de institución o modelo de seguro, tendencia política del gabinete, situación financiera del gobierno, globalización, importancia de importaciones y exportaciones en el producto, existencia de puntos de veto constitucionales y niveles de beneficio al inicio. Solo el efecto de la presencia de puntos de veto y de la globalización es débil y no significativo. En el caso de los niveles de beneficio al inicio el resultado es significativo pero de signo negativo, como si Tasas de Reemplazo más bajas representaran mayor espacio para retroceder. Combinaciones de tipos de gabinete con las demás variables permiten explorar el impacto bajo regímenes políticos diversos.
Scruggs ${ }^{1}$ construyó una serie de tiempo de 32 años-1971-2002- para los seguros de desempleo, enfermedad y pensiones de vejez para 18 países de la OECD y también un Índice de Beneficio Esperado a partir de la tasa de reemplazo y la cobertura poblacional. La tasa de reemplazo usada por Scru$\mathrm{ggs}^{21}$ corresponde al porcentaje que representan los beneficios en dinero menos impuestos a la renta fuera del trabajo, sobre el salario menos impuestos a la renta en el trabajo -incluyendo la contribución a la seguridad social-. La cobertura corresponde al porcentaje de personas que reciben el beneficio sobre el total de la fuerza laboral, en el caso de los seguros y respecto del total de personas en edad de retiro, en el caso de pensiones. El índice corresponde a la suma del producto de ambas tasas. El autor despliega información para los años 1972, 1987 y 2002 y países agrupados según la taxonomía clásica de regímenes de seguridad social: liberales, conservadores y socialdemócratas. La Tabla 3 muestra el índice para los años seleccionados y las tasas de reemplazo y cobertura del seguro de enfermedad.

De entre los años seleccionados, 1987 repre-

Tabla 3. Índice de beneficios esperado global y tasa de reemplazo y cobertura del seguro de enfermedad para 18 países de la OECD

\begin{tabular}{|c|c|c|c|c|c|c|c|c|c|}
\hline \multirow[b]{2}{*}{ Liberales } & \multicolumn{3}{|c|}{$\begin{array}{c}\text { Tasa de reemplazo del } \\
\text { seguro de enfermedad } \\
\text { (Porcentaje) }\end{array}$} & \multicolumn{3}{|c|}{$\begin{array}{l}\text { Tasa de cobertura del } \\
\text { seguro de enfermedad } \\
\text { (Porcentaje) }\end{array}$} & \multicolumn{3}{|c|}{$\begin{array}{l}\text { Índice de beneficio } \\
\text { esperado global }\left(^{*}\right) \\
\text { Máximo }=3\end{array}$} \\
\hline & 1972 & 1987 & 2002 & 1972 & 1987 & 2002 & 1972 & 1987 & 2002 \\
\hline Promedio & 37 & 50 & 46 & 74 & 74 & 68 & 1,24 & 1,61 & 1,55 \\
\hline Desviación St. & 28,1 & 24,5 & 25,0 & 34,0 & 33,5 & 39,2 & 0,47 & 0,42 & 0,32 \\
\hline Coef. Variación & 0,75 & 0,49 & 0,55 & 0,46 & 0,46 & 0,58 & 0,38 & 0,26 & 0,21 \\
\hline Conservadores & 1972 & 1987 & 2002 & 1972 & 1987 & 2002 & 1972 & 1987 & 2002 \\
\hline Promedio & 77 & 83 & 81 & 82 & 86 & 83 & 1,78 & 2,20 & 2,17 \\
\hline Desviación St. & 15,4 & 13,3 & 11.5 & 12.0 & 13,0 & 10,7 & 0,29 & 0,27 & 0,11 \\
\hline Coef. Variación & 0,20 & 0,16 & 0.14 & 0.15 & 0,15 & 0,13 & 0,16 & 0,12 & 0,05 \\
\hline S-Demócratas & 1972 & 1987 & 2002 & 1972 & 1987 & 2002 & 1972 & 1987 & 2002 \\
\hline Promedio & 76 & 88 & 78 & 95 & 97 & 96 & 2,09 & 2,58 & 2,40 \\
\hline Desviación St. & 12,1 & 9,8 & 14,0 & 7,1 & 4,8 & 5,0 & 0,19 & 0,25 & 0,25 \\
\hline Coef. Variación & 0,16 & 0,11 & 0,18 & 0,08 & 0,05 & 0,05 & 0,09 & 0,10 & 0,10 \\
\hline Total 18 países & 1972 & 1987 & 2002 & 1972 & 1987 & 2002 & 1972 & 1987 & 2002 \\
\hline Promedio & - & - & - & 82 & 84 & 80 & 1,63 & 2,04 & 1,95 \\
\hline Desviación St. & - & - & - & 24,6 & 24,7 & 28,6 & 0,51 & 0,53 & 0,46 \\
\hline Coef. Variación & - & - & - & 0,30 & 0,30 & 0,36 & 0,31 & 0,26 & 0,23 \\
\hline
\end{tabular}

(*) Incluye seguro de desempleo, de enfermedad y pensión de vejez. Fuente: Scruggs, L. The Generosity of Social Insurance, 197-2002. Oxford Review of Economics Policy. 22(2006):349-364. Extractado de Tablas 1, 2 y 3. 
senta el mejor momento para los indicadores y categorías de países. El 2002 suele mostrar una peor situación, aunque más cercana al año 1987 que al año 1972 en el caso del indicador global, salvo en el seguro de enfermedad.

La tasa de reemplazo suele ser, en todos los casos, menos generosa que la de cobertura y la situación base en el seguro de enfermedad es peor en los regímenes liberales. En el caso de los países liberales la tasa de reemplazo es significativamente más baja y la tasa más alta no se observa en los regímenes socialdemócratas, sino en los conservadores.

Los países con régimen socialdemócrata han ajustado preferentemente la tasa de reemplazo del seguro, mientras que los liberales han ajustado más la cobertura. Los países con regímenes conservadores han ajustado ambas en forma moderada.

En general, este conjunto de países de la OECD ha experimentado un retroceso en la generosidad de sus programas globales de seguridad social -seguros de cesantía y enfermedad más pensiones de vejez- pero continúan mejor que lo observado en 1972. Al decir de Bonato y Lusinyan ${ }^{22}$, los niveles de ausentismo en países de la OECD no son altos -índice promedio de 2,7 frente a un 2,6 observado en Estados Unidos-y han sido estables en las últimas décadas, pero con variaciones entre países y tasas altas en Holanda, Suecia, Noruega y Reino Unido. En el caso holandés, las reformas realizadas transfiriendo a los empleadores los costos del seguro han tenido significativo impacto en el ausentismo. La Tabla 4 muestra el gasto de los países en beneficios por ausencia al trabajo por enfermedad, en dos momentos.

Bonato y Lusinyan ${ }^{22}$ realizan un estudio econométrico para el Fondo Monetario Internacional, con información de los 18 países de la OECD usados por Scruggs. Los autores identifican variables que influirían en las tasas de ausentismo y las modelan. Estas variables son: la tasa de ausentismo (variable dependiente), la participación en la fuerza laboral, la estructura etárea, la expectativa de vida, el tamaño de la jornada laboral, el empleo part-time, el empleo por horas, la brecha de desempleo, la tasa de reemplazo del beneficio de enfermedad según Scruggs ${ }^{21}$, el índice de generosidad del beneficio de enfermedad según Scruggs ${ }^{21}$, la tasa de reemplazo del seguro de desempleo según Scruggs ${ }^{21}$, el índice de generosidad del seguro de desempleo según Scruggs ${ }^{21}$, la densidad de sin- dicatos como proxy del grado de protección del empleo, el índice de protección del empleo, la enfermedad pagada por los empleadores y el género.

La existencia de beneficio de enfermedad y el grado de generosidad del mismo muestran gran impacto en las tasas de ausentismo. Este fenómeno se explicaría por Moral Hazard.

El comportamiento del ausentismo frente al desempleo en este estudio no es contra-cíclico, contra lo esperado dada la selección de empleados que se realiza -se despide a los ausentistas- y el temor a perder el empleo que modera el comportamiento ausentista. Mayor ausentismo asociado a desempleo podría obedecer a mayor presión en el trabajo y mayor protección. Habría mayor ausentismo cuando la proporción de mujeres en

\section{Tabla 4. Gasto del gobierno en beneficios por ausencia al trabajo por enfermedad -Sickness- (como \% del PGB)}

\begin{tabular}{|lcc|}
\hline Países OECD & $\mathbf{1 9 8 0 - 8 9}$ & $\mathbf{1 9 9 0 - 9 9}$ \\
\hline Holanda & 2,18 & 1,55 \\
\hline Suecia & 2,19 & 1,39 \\
\hline Noruega & 1,53 & 1,35 \\
\hline España & 0,82 & 1,11 \\
\hline Irlanda & 1,68 & 0,90 \\
\hline Grecia & 0,16 & 0,79 \\
\hline Luxemburgo & 0,75 & 0,74 \\
\hline Dinamarca & 1,38 & 0,66 \\
\hline Finlandia & 0,53 & 0,55 \\
\hline Portugal & 0,53 & 0,55 \\
\hline Francia & 0,57 & 0,54 \\
\hline Suiza & 0,26 & 0,52 \\
\hline Italia & 0,32 & 0,47 \\
\hline Bélgica & 0,66 & 0,44 \\
\hline Alemania & 0,36 & 0,41 \\
\hline Estados Unidos & 0,25 & 0,24 \\
\hline Austria & 0,22 & 0,21 \\
\hline Reino Unido & 0,15 & 0,19 \\
\hline Islandia & $\mathrm{s} / \mathbf{1}$ & 0,09 \\
\hline
\end{tabular}

Fuente: Bonato, L. \& Lusinyan, L. Work Absence in Europe. International Monetary Found Working Paper. European Departament. 2004. WP/04/193. Basado en OECD, Social Expenditures Database. 
Tabla 5. Seguro de incapacidad laboral por enfermedad: comparación entre países

\begin{tabular}{|c|c|c|c|}
\hline & $\begin{array}{l}\text { Porcentaje del Ingreso a } \\
\text { pagar (tasa de reemplazo) }\end{array}$ & Período de espera & Duración máxima \\
\hline Alemania & $\begin{array}{l}100 \% \text { primeras } 6 \text { semanas y } \\
\text { después } 70 \% \text {. }\end{array}$ & $\begin{array}{l}\text { No hay. (Primeras } 6 \text { semanas son } \\
\text { de cargo del empleador) }\end{array}$ & 78 semanas en 3 años \\
\hline Argentina & $100 \%$ & No hay & 26 semanas \\
\hline Australia & $\begin{array}{l}100 \% \text { por } 5 \text { días o más según } \\
\text { ocupación (acumulables) y des- } \\
\text { pués Sickness Allowance }\end{array}$ & $\begin{array}{l}\text { No hay. (Pago de los } 5 \text { o más } \\
\text { primeros días son de cargo del } \\
\text { empleador) }\end{array}$ & \\
\hline Austria & $\begin{array}{l}100 \% \text { en las } 6 \text { a } 12 \text { primeras } \\
\text { semanas, } 50 \%+50 \% \text { las cuatro } \\
\text { siguientes, luego } 50 \% \text { y a partir } \\
\text { del día } 43,60 \% \text { con tope de } \\
3.450 \text { euros al mes }\end{array}$ & $\begin{array}{l}3 \text { días. (Empleador paga las } \\
\text { primeras } 6 \text { a } 12 \text { semanas y } 50 \% \\
\text { de las } 4 \text { siguientes) }\end{array}$ & 52 semanas extendible a 78 \\
\hline Bélgica & $\begin{array}{l}100 \% \text { a empleados los } 30 \text { pri- } \\
\text { meros días y luego } 60 \% .100 \% \\
\text { a obreros la primera semana y } \\
\text { luego } 60 \%\end{array}$ & $\begin{array}{l}1 \text { día. (Empleador paga a em- } \\
\text { pleados los primeros } 30 \text { días y a } \\
\text { obreros los primeros 15) }\end{array}$ & Un año. \\
\hline Bolivia & $75 \%$ & No hay & 26-52 semanas \\
\hline Brasil & $80-92 \%$ & No hay & No hay \\
\hline Colombia & $66.6 \%$ & 4 días & 180 días \\
\hline Costa Rica & $60 \%$ & No hay & 52 semanas \\
\hline Cuba & $50-90 \%$ & No hay & 11 semanas \\
\hline Chile & $\begin{array}{l}100 \% \text {, con tope de unos } 2.160 \\
\text { euros al mes (valores de mone- } \\
\text { das al } 23 / 02 / 2011 \text { ) }\end{array}$ & $\begin{array}{l}3 \text { días en licencias menores de } \\
11 \text { días para empleados parti- } \\
\text { culares }\end{array}$ & No hay tope en la práctica \\
\hline Dinamarca & $\begin{array}{l}100 \% \text { con tope de algo más de } \\
400 \text { euros semanales }\end{array}$ & $\begin{array}{l}\text { No hay. (En el caso de trabajado- } \\
\text { res con empleador, éste paga las } \\
\text { primeras } 2 \text { semanas). }\end{array}$ & $\begin{array}{l}52 \text { semanas dentro de un perío- } \\
\text { do de } 18 \text { meses }\end{array}$ \\
\hline España & $\begin{array}{l}60 \% \text { hasta día } 15 \text { y } 75 \% \text { a partir } \\
\text { el día } 16\end{array}$ & $\begin{array}{l}3 \text { días. (Empleador paga desde } \\
\text { el día } 4 \text { al día 15) }\end{array}$ & 12 meses extendible a 18 \\
\hline Est. Unidos & 0 a $75 \%$, no obligatorio & $\begin{array}{l}\text { Variable, según compañía de } \\
\text { seguro }\end{array}$ & 52 semanas \\
\hline Francia & $\begin{array}{l}50 \% \text { hasta el día } 30 \text { con tope en } \\
41,3 \text { euros al día y } 66.6 \% \text { con } \\
\text { tope } 55,0 \text { euros al día si tiene } \\
3 \text { o más niños dependientes. A } \\
\text { partir del séptimo mes a } 51,49 \% \\
\text { y } 68,66 \% \text {, respectivamente }\end{array}$ & 3 días & $\begin{array}{l}12 \text { meses por períodos de tres } \\
\text { años consecutivos. En la práctica } \\
36 \text { meses }\end{array}$ \\
\hline Grecia & $\begin{array}{l}\text { La que resulta de } 13.5 \text { euros dia- } \\
\text { rios los primeros } 15 \text { días y } 24.9 \\
\text { euros diarios a partir del día } 16\end{array}$ & 3 días & $\begin{array}{l}720 \text { días como máximo si exister } \\
1.500 \text { días de aseguramiento } \\
\text { previo }\end{array}$ \\
\hline Guatemala & $66 \%$ & No hay & 26-39 semanas \\
\hline
\end{tabular}


Tabla 5. Seguro de incapacidad laboral por enfermedad: comparación entre países (continuación)

\begin{tabular}{|c|c|c|c|}
\hline & $\begin{array}{l}\text { Porcentaje del Ingreso a } \\
\text { pagar (tasa de reemplazo) }\end{array}$ & Período de espera & Duración máxima \\
\hline Holanda & $70 \%$ con tope 167 euros diarios & $\begin{array}{l}\text { No hay. (Empleador paga la } \\
\text { totalidad del beneficio cuando } \\
\text { se trata de su empleado) }\end{array}$ & $\begin{array}{l}52 \text { semanas si paga el emplea- } \\
\text { dor y } 104 \text { si paga el fondo social }\end{array}$ \\
\hline Irlanda & $\begin{array}{l}134.8 \text { euros semanales más } \\
89.8 \text { euros semanales por adulto } \\
\text { dependiente y } 16.8 \text { por niño } \\
\text { dependiente }\end{array}$ & 3 días & $\begin{array}{l}\text { Sin límite si ha completado } 260 \\
\text { semanas de contribuciones y } 52 \\
\text { semanas si la contribución está } \\
\text { entre } 39 \text { y } 260\end{array}$ \\
\hline Islandia & 9,47 euros diarios & 14 días & $\begin{array}{l}52 \text { semanas dentro de un perío- } \\
\text { do máximo de } 24 \text { meses }\end{array}$ \\
\hline Italia & $\begin{array}{l}50 \% \text { hasta el día } 20 \text {, luego } \\
66.6 \%\end{array}$ & $\begin{array}{l}3 \text { días. (El seguro social está } \\
\text { orientado a los obreros. Los } \\
\text { Empleadores siguen pagando el } \\
\text { salario a los empleados por un } \\
\text { máximo de } 90 \text { días) }\end{array}$ & 180 días \\
\hline Japón & $60 \%$ & 3 días & 6 meses \\
\hline Luxemburgo & $100 \%$ & $\begin{array}{l}\text { No hay. (El seguro social está } \\
\text { orientado a los obreros. Los } \\
\text { Empleadores siguen pagando el } \\
\text { salario a los empleados por un } \\
\text { máximo de } 90 \text { días) }\end{array}$ & 52 semanas \\
\hline México & $60 \%$ & 3 días & 52 semanas \\
\hline Noruega & $\begin{array}{l}100 \% \text { y } 75 \% \text { a trabajadores por } \\
\text { cuenta propia }\end{array}$ & $\begin{array}{l}16 \text { días en trabajadores por } \\
\text { cuenta propia. (Empleador } \\
\text { paga los primeros } 16 \text { días a sus } \\
\text { empleados) }\end{array}$ & $\begin{array}{l}260 \text { días para empleados y } \\
248 \text { días para trabajadores por } \\
\text { cuenta propia }\end{array}$ \\
\hline Perú & $100 \%$ & $\begin{array}{l}\text { No hay ( } 20 \text { días pagados por el } \\
\text { empleador) }\end{array}$ & 49 semanas \\
\hline Portugal & $\begin{array}{l}65 \% \text { hasta el día } 365 \text { y } 70 \% \text { en } \\
\text { adelante }\end{array}$ & $\begin{array}{l}\text { No hay. (El pago por parte de los } \\
\text { empleadores no está regulado) }\end{array}$ & 1.095 días \\
\hline Reino Unido & $\begin{array}{l}77 \text { euros o } 91 \text { euros por semana } \\
\text { los primeros } 28 \text { días según } \\
\text { pague el empleador o el seguro, } \\
\text { respectivamente. En adelante, } \\
91 \text { euros semanales y } 102 \text { euros } \\
\text { si se está en edad de pensio- } \\
\text { narse. }\end{array}$ & $\begin{array}{l}3 \text { días (El empleador paga entre } \\
\text { los días } 4 \text { y } 28 \text {, salvo a las rentas } \\
\text { bajo } 109 \text { euros). }\end{array}$ & $\begin{array}{l}52 \text { semanas con evaluación mé- } \\
\text { dica de la incapacidad el día } 28\end{array}$ \\
\hline Suecia & $\begin{array}{l}65 \% \text { los días } 2 \text { y } 3 \text { y } 80 \text { a partir } \\
\text { del cuarto día. } \%\end{array}$ & $\begin{array}{l}1 \text { día. (Empleador paga del día } \\
2 \text { al } 21 \text { ) }\end{array}$ & No hay \\
\hline Suiza & $\begin{array}{l}100 \% \text { durante las primeras tres } \\
\text { semanas. Luego, no es obliga- } \\
\text { torio. }\end{array}$ & $\begin{array}{l}3 \text { días. (Empleador paga durante } \\
\text { las primeras } 3 \text { semanas) }\end{array}$ & $\begin{array}{l}720 \text { días dentro de un período } \\
\text { de } 900 \text { días consecutivos en } \\
\text { seguros voluntarios }\end{array}$ \\
\hline Uruguay & $70 \%$ & 3 días & 12 meses \\
\hline Venezuela & $50-66.6 \%$ & 3 días & 52 semanas \\
\hline
\end{tabular}

Fuentes: elaboración propia basada en Bonato, L. \& Lusinyan (2004), PUC (1999), Rodríguez (2003), Superintendencia de Salud (2006). 
la fuerza laboral es mayor. Se asociarían a menor ausentismo la flexibilidad en la jornada, la buena salud de las personas y la transferencia de costos al empleador.

\section{Chile en el contexto Internacional}

Sin pretender ser concluyente, la Tabla 5 resume información proporcionada por fuentes secundarias, situando en el contexto internacional a nuestro país sobre la base de tres parámetros constitutivos del seguro: i) tasa de reemplazo (el porcentaje de renta que el seguro restituye); ii) período de espera (tiempo de reposo médico sin restitución de renta) y, iii) duración máxima (frontera con la invalidez permanente).

$\mathrm{Si}$ se considera que OECD incluye preferentemente países cuyos sistemas de seguridad social establecidos por ley se reconocen como "comprehensivos", en cuanto a la cobertura del seguro, Chile, cuyo sistema se basa en el empleo formal (como el alemán), se sitúa por debajo de los países que brindan protección universal sobre una base de ciudadanía (países escandinavos) y por sobre aquellos que lo hacen focalizadamente, en función de necesidades (Australia y Reino Unido). Respecto de los países de América Latina incluidos en la tabla para facilitar la referenciación y también del conjunto de aquellos con sistemas "comprehensivos" establecidos, Chile se perfila como el que brinda una mejor protección en la materia, después de Argentina.

\section{Referencias}

1. Aedo C, Bastías G, Sapelli C. Subsidio de Incapacidad Laboral en Chile. Mimeo. 1999.

2. Briner, R. Absence From Work. ABC of Work Related Desorders 1996; 313: 874-7

3. Cid C. Microeconomía del Subsidio por Incapacidad laboral: Evaluación y Propuestas de Políticas sobre la base de un modelo con presencia de Riesgo Moral. Monografía de Graduación para obtener el grado de Master of Arts in Economics. Programa de Post-grado en Economía Ilades-Geogetown University. Mayo del 2002.

4. International Labour Organization (OIT): World Social Security Report 2010/11: providing coverage in times of crisis and beyond, Ginebra, 2010.
5. OECD. Reforming sickness and disability policies to improve work incentives. Department of Employment and Workplace Relations. Australian Governement. OECD Thematic Review, Country Note-Round Two. Noviembre 2006.

6. PUC. (Pontificia Universidad Católica de Chile, Instituto de Economía). Programa de Economía de la Salud. ¿Subsidios por Incapacidad Laboral versus Seguros de Salud? Marzo 1999.

7. Bowen A. Introducción a la Seguridad Social. Editorial Jurídica de Chile, Tercera edición actualizada, 1992.

8. Rodríguez J. y Tokman, M. Licencias Médicas ¿Chilenos Enfermizos?. En Foco No16. Corporación Expansiva. 2003.

9. Vergara, M. La Dicotomía Estado Mercado en el Sector de la Salud en Chile. Estudios Sociales, de la Corporación de Promoción Universitaria, No108, Semestre 2. 2001.

10. Castles, F. The Institutional Design of the Australian Welfare State. International Social Security Review. 1997; 50: 25-41.

11. Kangas O. Institutional Development of Sickness Cashbenefits Programmes in 18 OECD Countries. Social Policies and Administration 2004; 38 (2): 190-203.

12. Föster M. Sickness, Disability and Reintegration Strategies. OECD Directorate for Employment, Labour and Social Affairs. Social Policy Division. Santander, 16-20 July 2007.

13. Ministerio de Planificación, Gobierno de Chile. Chile Crece Contigo: Sistema de Protección Integral de la Primera Infancia. Secretaría Ejecutiva de Protección Social. Abril del 2007.

14. Instituto de Políticas Públicas y Gestión Salud y Futuro. Universidad Andrés Bello. Reforma de la salud en Chile: desafíos de la implementación. Sánchez H \& Labbé J, Editores. 2005.

15. Consejo Asesor Presidencial Trabajo y Equidad. Hacia un Chile más justo: Trabajo, Salario, Competitividad y Equidad Social. Informe Final. Mayo 2008.

16. Kangas O. Attitudes on Means-Tested Social Benefits in Finland. Acta Sociológica 1995; 38: 299-310.

17. Clasen J, Wim Van Oorschot. Changing Principles in European Social Security. European Journal of Social Security 2002; 4: 89-115.

18. Korpi W, Palme, J. New Politics and Class Politics in the Context of Austerity and Globalization: Welfare State Regress in 18 Countries, 1975-95. American Political Science Review 2003; 97: 425-46.

19. Pierson P. Three Worlds of Welfare State Research. Comparative Political Studies 2000; 33: 791-821.

20. Adema W. Revisiting Real Social Spending Across Coun- 
tries: a Brief Note. OECD Economics Studies 2000; 30: 192-7.

21. Scruggs, L. The Generosity of Social Insurance, 1972002. Oxford Review of Economics Policy 2006; 22:

\section{9-64.}

22. Bonato L, Lusinyan L. Work Absence in Europe. International Monetary Found Working Paper WP/04/193. European Departament. 2004. 